\title{
Cinética de Cristalização Não-isotérmica de Resíduos de Polietileno de Alta Densidade (PEAD)
}

\author{
Helson Moreira da Costa \\ Instituto Politécnico - IPRJ, Universidade do Estado do Rio de Janeiro - UERJ \\ Universidade Estácio de Sá - UNESA, Campus Nova Friburgo \\ Mônica Calixto de Andrade, Milena Diniz Lessa \\ Instituto Politécnico - IPRJ, Universidade do Estado do Rio de Janeiro - UERJ \\ Valéria Dutra Ramos \\ Universidade Estácio de Sá - UNESA, Campus Nova Friburgo
}

\begin{abstract}
Resumo: Nesta investigação, diversos resíduos incolores ou coloridos pós-consumo de polietileno de alta densidade (PEAD) foram caracterizados através do índice de fluidez (MFI) e da calorimetria exploratória diferencial (DSC). A equação de Avrami modificada por Jeziorny, e os modelos de Kissenger e Friedman foram utilizados para descrever a cinética de cristalização não-isotérmica das amostras. Foi verificado que os pigmentos presentes no PEAD têm diferentes habilidades nucleantes durante o processo de cristalização, bem como diferentes efeitos sobre a taxa de cristalização e a energia de ativação necessária para o transporte de segmentos macromoleculares para a superfície do cristal em crescimento.
\end{abstract}

Palavras-chave: Polietileno de alta densidade, análise térmica, cinética, cristalização.

\section{Non-isothermal Crystallization kinetics of High-density Polyethylene (HDPE) Scraps}

\begin{abstract}
In this study, miscellaneous uncolored and colored post-consumption high-density polyethylene (HDPE) scraps were characterized by melt flow index and differential scanning calorimetry (DSC). Avrami equation modified by Jeziorny, Kissenger and Friedman methods were employed to describe the non-isothermal crystallization kinetics of the samples. The colorants present in HDPE have different nucleating abilities during the crystallization process, as well as different effects upon the crystallization rate and the activation energy for the transport of macromolecular segments to the growing crystal surface.
\end{abstract}

Keywords: High-density polyethylene, thermal analysis, kinetics, crystallization.

\section{Introdução}

O consumo massivo de bens e serviços é uma das características da sociedade moderna. Ele se reflete na necessidade de um desenvolvimento industrial contínuo e na cobrança de um elevado preço do ponto de vista ambiental. Promulgada no dia 2 de agosto de 2010, após amplo debate com governo, universidades, setor produtivo e entidades civis, a Política Nacional de Resíduos Sólidos pode modificar profundamente o cenário. A lei lança uma visão moderna na luta contra um dos maiores problemas do planeta, o lixo urbano, tendo como princípio a responsabilidade compartilhada entre governo, empresas e população. A nova legislação impulsiona o retorno dos produtos às indústrias após o consumo e obriga o poder público a realizar planos para o gerenciamento do lixo ${ }^{[1,2]}$.

No entanto, o problema da reciclagem de resíduos, em especial a de materiais poliméricos, ainda não encontra uma solução satisfatória. Se, por um lado, a reciclagem primária possui mercado garantido uma vez que consiste na regeneração de um único tipo de resina separadamente e é, geralmente, associado à produção industrial (pré-consumo); por outro lado, a reciclagem secundária é um mercado cujo crescimento está condicionado ao encontro de novas tecnologias para processar os vários subtipos de materiais plásticos presentes rotineiramente no lixo urbano, particularmente poliolefinas - polietileno de alta densidade (PEAD), polietileno de baixa densidade (PEBD), polietileno linear de baixa densidade (PEBDL), e polipropileno (PP); poli(etileno tereftlato) (PET); poliestireno (PS); e, poli(cloreto de vinila) (PVC).

Entre os diversos problemas que podem ocorrer e que dificultam o processo de reciclagem secundária são citados $^{[3-7]}$ :

- Quando não existe a coleta seletiva, há a presença de contaminantes diversos junto aos resíduos plásticos - restos de comida, gorduras, papel, etiquetas, grampos e sujeira em geral. A consequência direta é a redução do preço de venda e a necessidade de morosas operações de lavagem e secagem;

- Mesmo quando há coleta seletiva, os resíduos plásticos são variados e necessitam ser classificados quanto ao tipo de resina. Ainda que exista uma padronização numérica por parte dos fabricantes

Autor para correspondência: Helson Moreira da Costa, Instituto Politécnico - IPRJ, Universidade do Estado do Rio de Janeiro - UERJ, Rua Bonfim, n. 25/parte, Vila Amélia, CEP 28625-570, Nova Friburgo, RJ, Brasil, e-mail: hmcosta@iprj.uerj.br 
(NBR 13.230 - Reciclabilidade e identificação de materiais plásticos, da Associação Brasileira de normas Técnicas - ABNT), o que facilita a identificação, há problemas de: mistura de resinas sem a identificação individual na embalagem, presença de pigmentos variados (embalagens coloridas), aditivos diversos, cargas, entre outros;

- A adequação da resina reciclada para uma determinada aplicação depende da demanda desta aplicação e da natureza da contaminação resultante do uso anterior da embalagem. Somado a isso, as resinas virgens são fabricadas com propriedades específicas (grades) de acordo com as necessidades da aplicação final.

Para o transformador que utilizará o material reciclado é importante que o uso total ou parcial do mesmo não afete negativamente a qualidade do artefato ou o desenvolvimento do processo de produção, condições que ocasionam perdas, refugos e oneram os custos produtivos. Assim, quanto melhor o processo de reciclagem e melhor a caracterização do resíduo plástico, maior será o valor agregado e mais alto o seu preço de mercado.

Deste modo, nesta investigação, embalagens pós-consumo de polietileno de alta densidade (PEAD) foram coletadas e, após a devida limpeza, foram caracterizadas através da técnica de calorimetria exploratória diferencial (DSC). O principal objetivo foi verificar a cinética de cristalização não-isotérmica e parâmetros como, por exemplo, as temperaturas de cristalização $\left(\mathrm{T}_{\mathrm{c}}\right)$, e fusão $\left(\mathrm{T}_{\mathrm{m}}\right)$ e o grau de cristalinidade $\left(\chi_{\mathrm{c}}\right)$, conforme o tipo de embalagem de PEAD analisada.

\section{Experimental}

\section{Material}

Os resíduos de PEAD foram escolhidos em função do quantitativo percentual em massa verificado na Central de Coleta Seletiva da Empresa Brasileira de Meio Ambiente (EBMA) situada na cidade de Nova Friburgo, região serrana do estado do Rio de Janeiro.
Embalagens coloridas ou não, de tamanhos e formatos diversos, foram selecionadas baseando-se na codificação numérica. Nesse código, os símbolos são constituídos por um elemento comum, formado por três setas inseridas em um triângulo e apontadas em sentido horário, e um código numérico - valor 2 para o PEAD.

Após a coleta, as embalagens foram lavadas em água corrente com detergente líquido. Tampas, rótulos e resíduos de cola foram retirados. Posteriormente, as embalagens foram secas ao ar e granuladas. Os grânulos foram mantidos por 30 minutos em contato com uma solução $1 \%$ de hidróxido de sódio conforme procedimento descrito por Remédio e colaboradores ${ }^{[6]}$. Após enxague até $\mathrm{pH}$ neutro, os grânulos foram separados por cor e colocados em estufa para secagem. Finalmente, através do procedimento de partição, os grânulos foram dispostos para as análises. O índice de fluidez (MFI) das amostras de PEAD foi determinado segundo a norma ASTM D1238, usando-se o equipamento Melt Flow Quick Index, marca Instron (Ceast Division).

Na Tabela 1 são apresentadas as embalagens de PEAD selecionadas nesta investigação, assim como a codificação experimental escolhida para a apresentação dos resultados.

Vale ressaltar que as embalagens de PEAD podem apresentar variadas colorações em função dos pigmentos orgânicos e inorgânicos disponíveis comercialmente; assim, as embalagens coloridas apresentadas neste trabalho foram as mais comuns encontradas como resíduo pós-consumo na Central de Coleta Seletiva de Nova Friburgo. Além disso, a escolha do pigmento para ser aplicado ao polímero não depende somente da cor e brilho, mas existem várias outras características que devem ser levadas em consideração para a seleção do pigmento apropriado. Em geral, os fabricantes utilizam um masterbatch (concentrado sólido até 30\% para pigmentos orgânicos ou até $80 \%$ para pigmentos inorgânicos) de cor, o qual é composto pelo colorante, polímero e aditivos (auxiliares de processo e promotores de propriedades específicas no produto final).

Tabela 1. Embalagens de polietileno de alta densidade (PEAD) escolhidas para análise de DSC.

\begin{tabular}{|c|c|c|c|c|c|}
\hline Embalagem & Cor/Agente ${ }^{1}$ & Visual $^{2}$ & Uso & Código & $\begin{array}{c}\text { Índice de fluidez } \\
(\mathrm{g} / 10 \mathrm{~min})\end{array}$ \\
\hline Produto de limpeza & $\begin{array}{l}\text { Pigment Green } 7 / f t a l o c i a n i n a \\
\text { de cobre, clorada }\end{array}$ & Verde & Alvejante & $\mathrm{Vd}$ & $1,61 \pm 0,06$ \\
\hline Produto de limpeza & Pigment White $6 / \mathrm{TiO}_{2}$, rutilo & Branco & Álcool comum & $\mathrm{Br}$ & $1,69 \pm 0,27$ \\
\hline Produto de limpeza & ---- & Incolor $^{3}$ & Amaciante & Inc & $1,27 \pm 0,18$ \\
\hline Produto de limpeza & Pigment Yellow 83/diarílico & Laranja & Desengordurante & $\mathrm{Lj}$ & $1,68 \pm 0,27$ \\
\hline Produto de limpeza & $\begin{array}{l}\text { Pigment Blue 15:0/Ftalocianina } \\
\text { de cobre, cristalização alfa }\end{array}$ & Azul escuro & Multiuso & AzEsc & $1,46 \pm 0,54$ \\
\hline Produto de limpeza & $\begin{array}{l}\text { Pigment Red 57:1/monoazoico } \\
\text { laqueado com } \mathrm{Ca}\end{array}$ & Rosa & Amaciante & Rs & $1,31 \pm 0,07$ \\
\hline Produto de higiene & $\begin{array}{l}\text { Pigment Red 170/monoazoico } \\
\text { derivado de Naftol AS }\end{array}$ & Vinho & Xampu & Vin & $1,14 \pm 0,11$ \\
\hline Produto de limpeza & Pigment Violet 23/dioxazina & Roxo & Multiuso & Rx & $1,02 \pm 0,10$ \\
\hline Produto automotivo & $\mathrm{CaCO}_{3}$ & Marfim & Lubrificante & Mrf & $1,09 \pm 0,10$ \\
\hline Produto de limpeza & $\begin{array}{l}\text { Pigment Blue } 15: 0 / \text { ftalocianina } \\
\text { de cobre, cristalização alfa }\end{array}$ & Azul claro & Amaciante & $\mathrm{AzCl}$ & $1,90 \pm 0,37$ \\
\hline
\end{tabular}




\section{Análise térmica}

Os resíduos de PEAD foram analisados através da técnica de calorimetria exploratória diferencial (DSC) utilizando-se o equipamento Perkin Elmer, modelo STA-6000, Silmutaneous Thermal Analyzer. Entre $20 \mathrm{mg}$ a $25 \mathrm{mg}$ de cada amostra foram usados. Cápsulas de porcelana foram utilizadas e, sob atmosfera de ar sintético, o procedimento de análise ocorreu mediante quatro ciclos simultâneos: (i) aquecimento da amostra da temperatura ambiente até $210{ }^{\circ} \mathrm{C}$ com uma taxa de $40{ }^{\circ} \mathrm{C} / \mathrm{min}$; (ii) Isoterma em $210{ }^{\circ} \mathrm{C}$ por 3 minutos; (iii) Resfriamento de $210^{\circ} \mathrm{C}$ até temperatura ambiente com taxas de 5, 10, 15 ou $20^{\circ} \mathrm{C} / \mathrm{min}$; (iv) Aquecimento da temperatura ambiente até $210{ }^{\circ} \mathrm{C}$ com taxas de 5, 10, 15 ou $20{ }^{\circ} \mathrm{C} / \mathrm{min}$. Antes de cada análise, para fins de calibração do equipamento, um padrão de índio (In) foi utilizado.

Parâmetros de interesse como a temperatura de cristalização ( $\mathrm{T}$ ); a temperatura de fusão $\left(\mathrm{T}_{\mathrm{m}}\right.$ - correspondente ao segundo aquecimento); a entalpia de cristalização $\left(\Delta \mathrm{H}_{c}-\right.$ medida da área sob a exoterma); a entalpia de fusão $\left(\Delta \mathrm{H}_{\mathrm{m}}\right.$ - medida da área sob a endoterma); o grau de cristalinidade $\left(\chi_{\mathrm{c}}-\right.$ calculado em relação ao PEAD 100\% cristalino); e, outros valores específicos para os modelos cinéticos escolhidos, foram obtidos a partir dos termogramas com auxílio do programa Pyris Thermal Analysis ${ }^{\mathrm{TM}}{ }^{\circledR}$, versão 10.1 .

\section{Resultados e Discussão}

\section{Índice de fluidez (MFI)}

Define-se o índice de fluidez (MFI) como sendo a quantidade em gramas de polímero, que flui durante dez minutos através de um orifício calibrado, em condições de força e de temperatura definidas. Quanto mais viscoso for o material no estado fundido, maior será sua massa molar média e menor será o valor do índice de fluidez. Na Tabela 1 são apresentados os valores de MFI das diferentes amostras de polietileno de alta densidade (PEAD).

A análise de variância (ANOVA) pode ser usada na investigação de situações nas quais existem diversas variáveis independentes. Quando são violadas de forma importante as pressuposições de normalidade e homocedasticidade, não se pode confiar no resultado de uma análise de variância tradicional, pois a probabilidade de se cometer um erro do Tipo I afasta-se marcadamente de $\alpha$. A alternativa não-paramétrica para a ANOVA a um critério é o teste de Kruskal-Wallis. O teste de KruskalWallis é o teste não-paramétrico utilizado na comparação de três ou mais amostras independentes. Ele nos indica se há diferença entre pelo menos dois deles. A aplicação do teste utiliza os valores numéricos transformados em postos e agrupados em um só conjunto de dados. A comparação dos grupos é realizada por meio da média dos postos (posto médio) ${ }^{[9,10]}$. Desta forma, com auxílio dos programas SigmaPlot ${ }^{\mathrm{TM} \otimes} 12.3$ e Minitab ${ }^{\mathrm{TM}}{ }^{\circledR} 16$, o teste $H$ de Kruskal-Wallis foi conduzido.

Para a análise dos resultados do MFI, as hipóteses estatísticas assumidas foram:

$$
\mathrm{H}_{\mathrm{o}}: \mathrm{MFI}_{\mathrm{Vd}}=\mathrm{MFI}_{\mathrm{Br}}=\mathrm{MFI}_{\mathrm{Inc}}=\ldots=\mathrm{MFI}_{\mathrm{Mrf}}
$$

$\mathrm{H}_{1}=\mathrm{H}_{\mathrm{o}}$ é falsa

As diferenças encontradas entre os valores médios de MFI das amostras de PEAD não foram grandes o suficiente para excluir a probabilidade de serem atribuídas a uma variabilidade aleatória, ou seja, não há diferença estatística significativa em um nível de confiança de $95 \%$ ( $H=13,83$ com 9 graus de liberdade, $\mathrm{p}=0,129)$. Assim sendo, ainda que as características exatas das diferentes resinas de PEAD não sejam conhecidas, pode-se inferir, mediante o resultado do teste não-paramétrico, que não há diferença relevante no comportamento viscoso das mesmas ou, indiretamente, em suas massas moleculares médias.

\section{Análise térmica}

Na Tabela 2 são apresentados os valores encontrados, a partir dos termogramas, para diferentes parâmetros das amostras de PEAD. De um modo geral, todas as amostras apresentam as mesmas tendências com o aumento das taxas de resfriamento/aquecimento:

- Há diminuição na temperatura de cristalização $\left(T_{c}\right.$, estimada pelo pico da curva exotérmica), na entalpia de cristalização $\left(\Delta \mathrm{H}_{\mathrm{c}}\right)$, na entalpia de fusão $\left(\Delta \mathrm{H}_{\mathrm{m}}\right) \mathrm{e}$ no grau de cristalinidade $\left(\chi_{\mathrm{c}}\right)$;

- Há um deslocamento dos picos das curvas endotérmicas, relativos à temperatura de fusão $\left(\mathrm{T}_{\mathrm{m}}\right)$, para valores mais elevados;

- Em taxas superiores a $5 \%$ min, há uma redução contínua das diferenças encontradas entre as amostras.

De acordo com Huang et al. ${ }^{[11]}$ e Zhang et al. ${ }^{[12]}$ um resfriamento mais rápido concede um menor tempo para a nucleação e o crescimento dos cristais, ou seja, há formação de um menor número de núcleos de crescimento com a consequentemente redução no número de cristais formados - redução dos valores de $\chi_{c}$ e dos parâmetros associados. Isso explica as tendências observadas e define, dentro das condições de análise, a taxa de $5{ }^{\circ} \mathrm{C} / \mathrm{min}$ como a taxa ótima para uma efetiva investigação das diferenças entre as amostras.

O PEAD-Inc apresenta os maiores valores de cristalinidade, sobretudo para taxas de resfriamento até $10{ }^{\circ} \mathrm{C} / \mathrm{min}$, enquanto a amostra PEAD-Mrf demonstra os menores valores de $\chi_{c}$. Por sua vez, PEAD-Rs e PEAD-Lj demonstram comportamento singular com pouca variação de cristalinidade - 5,5\% e 7,6\% de redução, respectivamente - conforme a taxa de resfriamento aumenta de $5 \%$ min para $20 \%$ min. Tais observações são ratificadas quando os dados de entalpia de cristalização $\left(\Delta \mathrm{H}_{\mathrm{c}}\right)$ e, sobretudo, os de entalpia de fusão $\left(\Delta \mathrm{H}_{\mathrm{m}}\right)$ são avaliados.

Quanto a temperatura de cristalização $\left(\mathrm{T}_{c}\right)$, pouca variação entre as amostras é observada em taxas de resfriamento até $10^{\circ} \mathrm{C} / \mathrm{min}$; para taxas maiores, PEAD-Rs apresenta uma redução atenuada em $\mathrm{T}_{\mathrm{c}}$, o que condiz com a pouca variação de $\chi_{c}$ encontrada.

Uma vez que os valores de MFI das diferentes amostras não revelam do ponto de vista estatístico 
Tabela 2. Parâmetros obtidos a partir dos termogramas para as diferentes amostras de PEAD em diferentes taxas de aquecimento/ resfriamento.

\begin{tabular}{|c|c|c|c|c|c|c|}
\hline \multirow[b]{2}{*}{ Amostra } & \multicolumn{5}{|c|}{ Parâmetros } & \multirow{2}{*}{$\begin{array}{c}\text { Taxa de } \\
\text { aquecimento// } \\
\text { resfriamento } \\
\left({ }^{\circ} \mathrm{C} / \mathrm{min}\right) \\
\end{array}$} \\
\hline & $\begin{array}{c}\mathbf{T}_{\mathrm{c}} \\
\left({ }^{\circ} \mathbf{C}\right)\end{array}$ & $\mathrm{T}_{\mathrm{m}}\left({ }^{\circ} \mathrm{C}\right)$ & $\Delta \mathbf{H}_{\mathrm{c}}(\mathrm{J} / \mathrm{g})$ & $\Delta \mathbf{H}_{\mathrm{m}}(\mathrm{J} / \mathrm{g})$ & $\chi_{\mathrm{c}}{ }^{1}$ & \\
\hline \multirow[t]{4}{*}{ Verde } & 115,7 & 137,0 & 250,5 & 192,7 & 65,8 & 5 \\
\hline & 110,5 & 140,1 & 213,8 & 179,5 & 61,3 & 10 \\
\hline & 107,3 & 142,6 & 203,8 & 172,3 & 58,8 & 15 \\
\hline & 105,0 & 147,7 & 171,6 & 155,4 & 53,0 & 20 \\
\hline \multirow[t]{4}{*}{ Branco } & 114,7 & 139,3 & 214,5 & 183,0 & 62,5 & 5 \\
\hline & 112,0 & 140,1 & 203,1 & 166,4 & 56,8 & 10 \\
\hline & 105,4 & 145,8 & 187,9 & 154,0 & 52,5 & 15 \\
\hline & 104,7 & 146,2 & 144,3 & 141,3 & 48,2 & 20 \\
\hline \multirow[t]{4}{*}{ Incolor } & 116,9 & 137,1 & 254,0 & 203,6 & 69,5 & 5 \\
\hline & 110,7 & 140,7 & 217,7 & 191,3 & 65,3 & 10 \\
\hline & 107,6 & 144,0 & 204,7 & 178,0 & 60,8 & 15 \\
\hline & 106,1 & 148,4 & 174,4 & 167,0 & 57,0 & 20 \\
\hline \multirow[t]{4}{*}{ Laranja } & 113,8 & 133,5 & 182,2 & 173,0 & 59,1 & 5 \\
\hline & 110,3 & 136,6 & 181,4 & 165,7 & 56,5 & 10 \\
\hline & 106,4 & 139,2 & 168,0 & 160,1 & 54,6 & 15 \\
\hline & 106,1 & 143,3 & 134,5 & 160,0 & 54,6 & 20 \\
\hline \multirow[t]{4}{*}{ Azul escuro } & 116,2 & 135,6 & 215,1 & 191,5 & 65,4 & 5 \\
\hline & 109,7 & 140,8 & 198,6 & 186,5 & 63,7 & 10 \\
\hline & 106,9 & 146,0 & 172,1 & 180,9 & 61,7 & 15 \\
\hline & 104,3 & 146,2 & 152,8 & 172,9 & 59,0 & 20 \\
\hline \multirow[t]{4}{*}{ Rosa } & 115,6 & 135,8 & 203,9 & 191,2 & 65,3 & 5 \\
\hline & 111,7 & 139,1 & 199,2 & 185,7 & 63,4 & 10 \\
\hline & 109,5 & 142,4 & 188,3 & 183,1 & 62,5 & 15 \\
\hline & 107,9 & 142,5 & 158,1 & 180,9 & 61,7 & 20 \\
\hline \multirow[t]{4}{*}{ Vinho } & 113,7 & 132,1 & 167,3 & 171,0 & 58,4 & 5 \\
\hline & 111,1 & 134,8 & 164,4 & 167,5 & 57,2 & 10 \\
\hline & 104,3 & 137,6 & 156,3 & 157,6 & 53,8 & 15 \\
\hline & 101,0 & 142,9 & 139,6 & 140,6 & 48,0 & 20 \\
\hline \multirow[t]{4}{*}{ Roxo } & 114,4 & 135,5 & 192,5 & 188,3 & 64,3 & 5 \\
\hline & 109,8 & 138,3 & 189,4 & 166,8 & 56,9 & 10 \\
\hline & 106,2 & 141,2 & 176,3 & 150,2 & 51,3 & 15 \\
\hline & 105,1 & 141,6 & 169,1 & 136,4 & 46,6 & 20 \\
\hline \multirow[t]{4}{*}{ Marfim } & 115,3 & 133,5 & 179,9 & 170,3 & 58,1 & 5 \\
\hline & 109,7 & 137,9 & 172,9 & 154,8 & 52,8 & 10 \\
\hline & 106,2 & 141,6 & 155,9 & 131,3 & 44,8 & 15 \\
\hline & 105,0 & 144,4 & 144,5 & 117,9 & 40,2 & 20 \\
\hline \multirow[t]{4}{*}{ Azul claro } & 114,5 & 135,7 & 195,9 & 179,9 & 61,4 & 5 \\
\hline & 109,3 & 140,4 & 185,9 & 163,5 & 55,8 & 10 \\
\hline & 105,5 & 143,7 & 181,7 & 151,8 & 51,8 & 15 \\
\hline & 103,6 & 144,7 & 165,5 & 137,7 & 47,0 & 20 \\
\hline
\end{tabular}

${ }^{1}$ Calculado de acordo com: $\chi_{\mathrm{c}}=\frac{\Delta \mathrm{H}_{\mathrm{m}}}{\Delta \mathrm{H}_{\mathrm{m}}^{0}} \times 100$, onde $\Delta \mathrm{H}_{\mathrm{m}}{ }^{\circ} \mathrm{p} / \mathrm{PEAD} 100 \%$ cristalino $=293 \mathrm{~J} / \mathrm{g}$.

diferenças relevantes, em um nível de confiança de 95\%, os comportamentos encontrados talvez possam ser atribuídos a distribuição de massa molecular das amostras de PEAD e ao efeito da concentração e da natureza dos colorantes e aditivos usados.

\section{Cinética de cristalização não-isotérmica}

A cristalização é um processo de transformação de fase que, do ponto de vista fenomenológico, é geralmente descrito por uma equação cinética generalizada. A maioria dos métodos que descrevem a cinética de cristalização de polímeros são baseados na equação de Avrami ${ }^{[13,14]}$, 
a qual assume que o grau de cristalinidade relativa, $\chi_{t}$, relaciona-se com o tempo de cristalização, em condições isotérmicas, através da Equação 1.

$$
\ln \left[-\ln \left(1-\chi_{\mathrm{t}}\right)\right]=\ln \mathrm{Z}_{\mathrm{t}}+\mathrm{n} \ln \mathrm{t}
$$

onde: $n$ é o índice de Avrami, uma constante que depende do tipo de nucleação e do processo de crescimento dos cristais; $Z_{t}$ é uma constante de taxa que envolve parâmetros associados a nucleação e ao crescimento; e, $\chi_{t}$ é a cristalinidade relativa, a qual em função da temperatura de cristalização é definida pela Equação 2.

$$
\chi_{\mathrm{t}}=\int_{\mathrm{T}_{\mathrm{o}}}^{\mathrm{T}}\left(\mathrm{dH}_{\mathrm{c}} / \mathrm{dT}\right) \mathrm{dT} / \int_{\mathrm{T}_{\mathrm{o}}}^{\mathrm{T}_{\infty}}\left(\mathrm{dH}_{\mathrm{c} / \mathrm{dT}}\right) \mathrm{dT}
$$

onde: $\mathrm{T}_{\mathrm{o}}$ e Too representam as temperaturas de início e de término do processo de cristalização, respectivamente. Contudo, em processos de importância prática, a cristalização ocorre sob condições não-isotérmicas e torna-se necessário obter uma relação mais apropriada.

Jeziorny ${ }^{[15]}$ sugere que a Equação 1 pode ser utilizada, desde que o parâmetro $Z_{t}$ e o tempo sejam corrigidos conforme a Equação 3, onde $\phi$ é a taxa de resfriamento:

$$
\ln Z_{c}=\ln Z_{t} / \phi \quad t=\left|T-T_{0}\right| / \phi
$$

A Tabela 3 apresenta os parâmetros encontrados a partir da aplicação do modelo de Jaziorny ${ }^{[15]}$. Os coeficientes de correlação são todos bastante próximos ou iguais a $1\left(\mathrm{r}^{2} \geq 0,99\right.$; exceção da amostra PEAD-Mrf resfriada em $5{ }^{\circ} \mathrm{C} /$ min cujo $\mathrm{r}^{2}=0,982$ ). Isto contradiz algumas investigaçõe s $^{[16,17]}$, onde perdas consideráveis na correlação linear são verificadas nos últimos estágios da cristalização, o que costuma dividir as curvas do modelo de Jaziorny em dois estágios com inclinações distintas atribuídos a cristalização primária e a cristalização secundária. Dentro das condições investigadas, o modelo parece indicar que o mecanismo de cristalização secundária ocorre apenas em pequena extensão, pois os coeficientes de correlação refletem uma dependência linear significativa.

O parâmetro $n$ não tem o mesmo significado físico que o determinado em condições isotérmicas, porém, de acordo com a literatura ${ }^{[18-21]}$, os valores do encontrados para as diferentes amostras de PEAD indicam que o tipo de nucleação e a forma de crescimento dos esferulitos são relativamente simples com formação de cristais tipo agulha a partir de núcleos esporádicos ou cristais em forma de disco a partir de núcleos instantâneos $(1<n$ $<2,5)$.

Para uma mesma amostra, o modelo de Jaziorny produziu retas aproximadamente paralelas umas as outras, o que indica que o mecanismo de cristalização e geometria de crescimento dos cristais ocorrem de forma similar. Além disso, os valores fracionários encontrados de $n$ são típicos de taxas de crescimento não lineares, as quais podem ser atribuídas a impurezas presentes (nucleação heterogênea) e/ou regimes de crescimento diferentes.
As amostras codificadas por PEAD-Vin, PEAD-Rx e PEAD-Mrf, por sua vez, exibem os menores valores de $n$, ou seja, demonstram uma nucleação e um processo de crescimento dos cristais mais simples e, desta forma, devem encontrar respaldo em valores de $\mathrm{T}_{\mathrm{m}}$ menores, $\mathrm{o}$ que também é confirmado pela Tabela 2.

$\mathrm{O}$ parâmetro $\mathrm{Z}_{\mathrm{c}}$ é um indicativo da taxa de cristalização e está condicionado ao mecanismo de nucleação e crescimento dos cristais. Novamente, para taxas de resfriamento superiores a $10{ }^{\circ} \mathrm{C} / \mathrm{min}$ pouca diferença é verificada entre as amostras. Contudo, em $5{ }^{\circ} \mathrm{C} / \mathrm{min}$, observa-se que PEAD-Br possui o menor valor de $Z_{c}$, ou seja, a cristalização da amostra ocorre da forma mais lenta. Se os cristais em PEAD-Br assumem uma geometria mais complexa e, possivelmente, são maiores conforme atestam os valores do expoente $n$ já discutidos, então, $Z_{c}$ apenas é um reflexo de tal processo.

Por sua vez, em $5{ }^{\circ} \mathrm{C} / \mathrm{min}$, os pares PEAD-Vin e PEAD-AzCl e PEAD-Rx e PEAD-Mrf têm valores próximos e elevados $Z_{c}$, ou seja, as amostras tem um processo global de cristalização facilitado, o que se reflete em cristais que crescem mais rápido, porém com geometria mais simples - como comprovam os valores de $n$ encontrados.

Além dos diferentes tipos de resinas de PEAD disponíveis no mercado para o processamento por injeção/sopro, as embalagens utilizadas neste estudo podem conter teores variados de pigmentos, aditivos e/ou cargas. Assim, atribuir à causas específicas as diferenças encontradas nos parâmetros do modelo de Jaziorny ${ }^{[15]}$ torna-se inviável.

No entanto, o índice de fluidez (MFI) das amostras, de acordo com a análise estatística, não apresentou diferenças significativas para um nível de confiança de $95 \%$. Além disso, a presença de aditivos (auxiliares de processamento, antioxidantes e outros) geralmente ocorre em percentuais pequenos. Desta forma, é possível inferir que os principais efeitos sobre os valores de $n$ e $\mathrm{Z}_{\mathrm{c}}$ possam ser derivados da presença de pigmentos ou cargas nos resíduos de PEAD e, assim, encontrar na literatura investigações que possam embasar os resultados experimentais obtidos.

De acordo com Ferreira ${ }^{[22]}$, os pigmentos orgânicos apresentam-se na forma de pequenos cristais aciculares, ou seja, na forma de pequenas agulhas (cristais primários da ordem de $0,05 \mu \mathrm{m}$ a $0,1 \mu \mathrm{m}$ ), as quais devido à elevada energia superficial e outros fatores formam aglomerados. Esses aglomerados podem, então, atuar como núcleos de cristalização gerando esferulitos cujo desenvolvimento acaba restrito pela elevada densidade de nucleação.

Em outro estudo, Broda ${ }^{[23]}$ investigando fibras de polipropileno incolores e pigmentadas com quinacridona e ftalocianina aponta que as fibras coloridas apresentam maior densidade de nucleação e esferulitos de menor tamanho, quando as condições de fiação usam baixas velocidades de estiramento. Além disso, a natureza do pigmento também tem influência na estrutura cristalina final.

Em processos de cristalização não-isotérmica, a energia de ativação pode ser estimada mediante o método 
Tabela 3. Parâmetros obtidos partir do modelo de Jeziorny ${ }^{[15]}$.

\begin{tabular}{|c|c|c|c|c|c|}
\hline \multirow{2}{*}{ Amostra } & \multicolumn{4}{|c|}{ Parâmetros } & \multirow{2}{*}{$\begin{array}{c}\text { Taxa de } \\
\text { aquecimento/ } \\
\text { resfriamento } \\
\left({ }^{\circ} \mathrm{C} / \mathrm{min}\right)\end{array}$} \\
\hline & $n$ & $Z_{t}\left(x 10^{4}\right)$ & $Z_{c}\left(x 1^{3}\right)$ & $\mathbf{r}^{2}$ & \\
\hline \multirow[t]{4}{*}{ Verde } & 1,60 & 3,50 & 4,20 & 0,993 & 5 \\
\hline & 1,90 & 1,82 & 1,03 & 1 & 10 \\
\hline & 2,02 & 1,72 & 0,69 & 1 & 15 \\
\hline & 2,31 & 0,91 & 0,27 & 0,998 & 20 \\
\hline \multirow[t]{4}{*}{ Branco } & 1,78 & 2,37 & 1,94 & 0,999 & 5 \\
\hline & 1,90 & 1,62 & 1,42 & 0,999 & 10 \\
\hline & 2,12 & 1,14 & 0,42 & 0,999 & 15 \\
\hline & 2,30 & 1,05 & 0,34 & 0,998 & 20 \\
\hline \multirow[t]{4}{*}{ Incolor } & 1,61 & 5,82 & 6,98 & 0,994 & 5 \\
\hline & 1,77 & 4,11 & 2,47 & 0,998 & 10 \\
\hline & 1,99 & 2,54 & 1,02 & 0,999 & 15 \\
\hline & 2,26 & 1,33 & 0,40 & 0,999 & 20 \\
\hline \multirow[t]{4}{*}{ Laranja } & 1,64 & 3,57 & 4,25 & 0,997 & 5 \\
\hline & 1,79 & 3,54 & 2,14 & 0,998 & 10 \\
\hline & 1,90 & 3,22 & 1,29 & 0,997 & 15 \\
\hline & 2,21 & 1,86 & 0,56 & 0,999 & 20 \\
\hline \multirow[t]{4}{*}{ Azul escuro } & 1,54 & 6,29 & 7,54 & 0,993 & 5 \\
\hline & 1,87 & 2,07 & 1,24 & 0,999 & 10 \\
\hline & 2,07 & 1,47 & 0,51 & 0,999 & 15 \\
\hline & 2,25 & 1,27 & 0,44 & 0,999 & 20 \\
\hline \multirow[t]{4}{*}{ Rosa } & 1,55 & 6,20 & 7,45 & 0,997 & 5 \\
\hline & 1,71 & 5,29 & 3,18 & 0,997 & 10 \\
\hline & 1,98 & 3,77 & 1,13 & 1 & 15 \\
\hline & 2,04 & 2,42 & 0,97 & 0,999 & 20 \\
\hline \multirow[t]{4}{*}{ Vinho } & 1,46 & 11,65 & 13,98 & 0,997 & 5 \\
\hline & 1,50 & 8,00 & 9,60 & 0,993 & 10 \\
\hline & 1,70 & 5,54 & 2,21 & 0,999 & 15 \\
\hline & 2,02 & 2,31 & 0,69 & 0,999 & 20 \\
\hline \multirow[t]{4}{*}{ Roxo } & 1,44 & 9,03 & 10,82 & 0,992 & 5 \\
\hline & 1,59 & 7,96 & 4,78 & 0,991 & 10 \\
\hline & 1,81 & 5,09 & 1,77 & 0,999 & 15 \\
\hline & 1,94 & 4,42 & 1,53 & 1 & 20 \\
\hline \multirow[t]{4}{*}{ Marfim } & 1,46 & 8,67 & 10,41 & 0,982 & 5 \\
\hline & 1,62 & 7,41 & 4,44 & 0,997 & 10 \\
\hline & 1,85 & 4,84 & 1,45 & 0,999 & 15 \\
\hline & 1,92 & 3,43 & 1,37 & 1 & 20 \\
\hline \multirow[t]{4}{*}{ Azul claro } & 1,41 & 11,40 & 13,68 & 0,997 & 5 \\
\hline & 1,64 & 6,02 & 3,61 & 0,999 & 10 \\
\hline & 1,80 & 4,75 & 1,90 & 0,999 & 15 \\
\hline & 1,95 & 4,42 & 1,32 & 1 & 20 \\
\hline
\end{tabular}

proposto por Kissenger ${ }^{[14]}$, o qual pode ser representado pela Equação 4 abaixo:

$$
\frac{\left[\ln \left(\frac{\phi}{T_{c}^{2}}\right)\right]}{\left(\frac{1}{T_{c}}\right)}=-\frac{E_{a}}{R}
$$

onde: R é a constante dos gases, 8,314 J.K-1.mol ${ }^{-1} ; \mathrm{e}, \mathrm{T}_{\mathrm{c}}$ é a temperatura referente ao pico da curva exotérmica de cristalização. A energia de ativação ( $\mathrm{E}_{\mathrm{a}}$ ) pode, então, ser calculada pela inclinação da reta obtida no gráfico $\ln$ $\left(\phi / \mathrm{T}_{\mathrm{c}}^{2}\right)$ versus $1 / \mathrm{T}_{\mathrm{c}}$.

Métodos de isoconversão podem, em princípio, serem aplicados em cristalizações não-isotérmicas para estimar a dependência da energia de ativação efetiva em relação à conversão $\mathrm{e}$ a temperatura ${ }^{[14]}$. $\mathrm{O}$ modelo matemático 


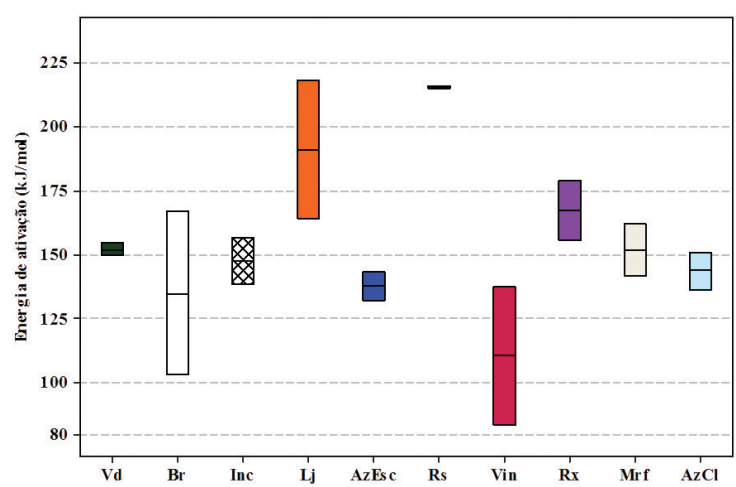

(a)

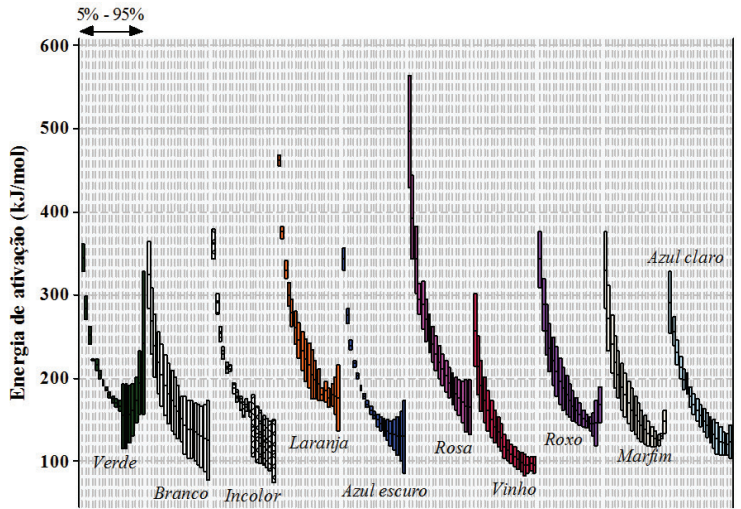

(b)

Figura 1. Energias de ativação para as diferentes amostras de PEAD. a) Equação de Kissenger ${ }^{[14]}$; b) Equação de Friedman ${ }^{[14,19]}$ para o intervalo de cristalinidade relativa de $5 \%$ a $95 \%$.

desenvolvido por Friedman ${ }^{[14,19]}$ permite o cálculo da energia de ativação para cada grau de cristalinidade segundo a Equação 5 abaixo:

$$
\ln \left(\frac{\mathrm{d} \chi_{\mathrm{t}}}{\mathrm{dt}}\right)_{\chi_{\mathrm{t}}}=\mathrm{C}-\Delta \mathrm{E} / \mathrm{RT}
$$

onde: $\mathrm{C}$ é um fator arbitrário pré-exponencial; $\Delta \mathrm{E}$ é a energia de ativação para um dado valor de cristalinidade relativa, $\chi_{\mathrm{t}} ; \mathrm{T}$ refere-se a uma série de temperaturas relacionadas a uma dada cristalinidade $\chi_{\mathrm{t}}$ em diferentes taxas de resfriamento; $\mathrm{R}$ é a constante universal dos gases $\left(8,314 \mathrm{~J} \mathrm{~mol}^{-1} \mathrm{~K}^{-1}\right)$; e, d $\chi_{t} / \mathrm{dt}$ é a taxa de cristalização instantânea em função do tempo para uma dada cristalinidade relativa $\chi_{\mathrm{t}}$. As temperaturas para determinado valor de $\chi_{\mathrm{t}}$ podem ser escolhidas selecionando-se graus de cristalinidade relativa - neste estudo, a faixa de $5 \%$ a $95 \%$ foi escolhida para as amostras de PEAD.

As Figuras 1a e $1 \mathrm{~b}$ apresentam os intervalos de valores estimados para as energias de ativação das amostras de PEAD usando as Equações de Kissenger ${ }^{[14]} \mathrm{e}$ Friedman ${ }^{[14,19]}$, respectivamente.

A energia de ativação calculada é a quantidade de energia mínima para se transportar segmentos das cadeias macromoleculares para a superfície dos cristais em crescimento. Para todas as amostras de PEAD, os valores de $E_{a}$ nos estágios iniciais do processo de cristalização das amostras são sempre elevados. Para valores de $\chi_{t}$ até $10 \%$, com exceção da amostra PEAD-Vin, os valores de $\mathrm{E}_{\mathrm{a}}$ são iguais ou superiores a $300 \mathrm{~kJ} / \mathrm{mol}$ (Figura 1b). Com o decorrer do processo, $\mathrm{E}_{\mathrm{a}}$ diminui progressivamente, voltando a crescer nos estágios finais $\left(\chi_{\mathrm{t}}=85 \%\right)$.

As amostras PEAD-Br, PEAD-Lje PEAD-Vin exibem coeficientes de correlação $\left(\mathrm{r}^{2}\right)$ de $0,850,0,942$ e 0,842 , respectivamente. $\mathrm{O}$ desvio da linearidade, proposta pela equação de Kissenger, produz um faixa de variação maior para os valores de $\mathrm{E}_{\mathrm{a}}$ global dessas amostras como mostra a Figura 1a. Além disso, observa-se que as amostras PEAD-Lj e PEAD-Rs são as que apresentam os maiores valores globais médios de $\mathrm{E}_{\mathrm{a}}$ com 191 e $215 \mathrm{~kJ} / \mathrm{mol}$, respectivamente. Em contrapartida, a amostra PEAD-Vin é a que exibe o menor valor, cerca de $110 \mathrm{~kJ} / \mathrm{mol}$.
Os baixos valores de $\mathrm{E}_{\mathrm{a}}$ apresentados pela amostra PEAD-Vin são condizentes com os elevados valores de $Z_{c}$ e baixos valores de $n$ e $\chi_{c}$ já discutidos. Possivelmente, uma das razões para tal comportamento possa ser a concentração do pigmento (aproximadamente $25 \%$ ) - uma maior densidade de núcleos pode facilitar o início do processo, mas acaba impedindo um crescimento efetivo dos esferulitos e reduzindo a cristalinidade.

As amostras PEAD-Rs e PEAD-Lj exibem valores de $Z_{c}$ de duas a três vezes menores que o valor de $Z_{c}$ da amostra PEAD-Vin, enquanto os valores de $n$ são maiores e $\chi_{c}$ da amostra PEAD-Rs é sempre o maior dentre as três. De acordo com informações do fabricante ${ }^{[8]}$, os pigmentos são usados em concentração de $15 \%$. Assim, após uma maior dificuldade inicial do processo de cristalização, atestado pelos valores de $\mathrm{E}_{\mathrm{a}}$ da Figura $1 \mathrm{~b}$, a nucleação heterogênea tomar lugar e os esferulitos podem assumir uma geometria mais complexa ( $n$ maior) em maior tempo $\left(\mathrm{Z}_{\mathrm{c}}\right.$ menor). Vários fatores podem afetar a ação de agentes denominados nucleantes em poliolefinas. Assim, talvez a melhor dispersão e a natureza do pigmento, além de uma eventual maior mobilidade das cadeias poliméricas, na amostra PEAD-Rs tenha permitido o maior valor de $\chi_{c}$ encontrado.

A análise de Kruskal-Wallis foi aplicada em conjunto com o método de Dunn ${ }^{[9,10]}$ aos dados da Figura $1 \mathrm{~b}$. Usando-se a amostra PEAD-Inc como grupo de controle $(\mathrm{H}=89,34, p \leq 0,001)$, os resultados revelam que PEAD-Vin, PEAD-Lj e PEAD-Rs são as únicas amostras que diferem de forma estatisticamente significativa (nível de confiança de $95 \%$ ), ou seja, os processo de cristalização do PEAD incolor ou com pigmentação, acompanhado mediante a equação de Friedman, transcorre com valores de $E_{a}$ equivalentes.

\section{Conclusões}

A heterogeidade da natureza das embalagens de PEAD - grade específico de resina utilizada, concentração exata do pigmento presente, presença de aditivos e/ou cargas - inviabiliza atribuir causas específicas às variações nos parâmetros cinéticos encontradas. No entanto, os resultados de índice de fluidez (MFI) não 
apresentaram diferenças significativas em um nível de confiança de 95\%; e, além disso, o teor de aditivos (auxilares de processamento, antioxidantes, antiestáticos e outros) é, em geral, pequeno. Assim sendo, foi inferido que o tipo colorante e/ou carga presente nas embalagens é o principal, ainda que não o único, agente responsável pelos efeitos observados.

A aplicação dos modelos de Kissenger e Friedman demonstrou que os processos de nucleação e crescimento dos cristais mostram-se energeticamente similares entre as amostras, apenas o PEAD-Vin, PEAD-Lj e o PEAD-Rs são estatisticamente diferentes da amostra de controle de PEAD-Inc (polietileno sem colorantes). Em contrapartida, a equação de Avrami modificada permitiu verificar que, dependendo do tipo de colorante encontrado, há diferenças importantes - os cristais adquirem uma geometria mais complexa (PEAD-Br) ou são formados com maiores valores de constante de taxa $\mathrm{Z}_{c}$ (PEAD-Vin, PEAD-Rx e PEAD-Mrf).

Como a microestrutura é um dos fatores que afeta o desempenho mecânico e o processamento dos artefatos, os dados experimentais encontrados ressaltam a atenção que deve ser dada durante o reaproveitamento de resíduos PEAD, particularmente, embalagens coloridas pós-consumo.

\section{Agradecimentos}

Os autores agradecem ao CNPq, CAPES e FAPERJ pelo suporte financeiro para o desenvolvimento do projeto.

\section{Referências Bibliográficas}

1. Compromisso Empresarial para Reciclagem - CEMPRE. Disponível em: <http://www.cempre.org.br>. Acesso em: maio 2013.

2. Empresa Brasileira de Meio Ambiente - EBMA. Disponível em: <http://www.ebma.com.br>. Acesso em: maio 2013.

3. Mano, E. B.; Pacheco, E. B. M. A. \& Bonelli, C. M. C. - "Meio ambiente, poluição e reciclagem", Edgard Blücher, São Paulo (2005).

4. Brognoli, R. - "Desenvolvimento da qualidade na reciclagem de resíduos plásticos", Senai, Porto Alegre (2006). (Dossiê Técnico).

5. Grippi, S. - "Lixo: reciclagem e sua história", Interciência, Rio de Janeiro (2006). PMCid:PMC1544341

6. Remédio, M. V. P.; Zanin, M. \& Teixeira, B. A. N. - Polímeros, 9, p.177 (1999). http://dx.doi.org/10.1590/ S0104-14281999000400029

7. Candian, L. M. - "Estudo do polietileno de alta densidade reciclado para uso em elementos estruturais", Dissertação de Mestrado, Escola de Engenharia de São Carlos, Universidade de São Paulo, Brasil (2007).

8. Tríplice Cor. Disponível em: <http://www.triplicecor.com. br>. Acesso em: jul. 2013.

9. Field, A. - "Descobrindo a estatística usando o SPSS", Bookman, Porto Alegre (2009).

10. Witte, R. S. \& Witte, J. S. - "Estatística", LTC, Rio de Janeiro (2005).

11. Huang, Y. P.; Chen, G. M.; Yao, Z.; Li, H. W. \& Wu, Y. - Eur. Polym. J., 41, p.2753 (2005). http://dx.doi.org/10.1016/j. eurpolymj.2005.02.034

12. Zhang, J.; Chen, S.; Su, J.; Shi, X.; Jin, J.; Wang, X. \& Xu, Z.- J. Therm. Anal. Calorim., 97, p.959 (2009). http:// dx.doi.org/10.1007/s10973-009-0014-7

13. Ozawa, T. - Polymer, 12, p.150 (1971). http://dx.doi. org/10.1016/0032-3861(71)90041-3

14. Di Lorenzo, L. \& Silvestre C. - Prog. Polym. Sci., 24, p.917 (1999). http://dx.doi.org/10.1016/S0079-6700(99)00019-2

15. Saengsuwan, S.; Tongkasee, P.; Sudyoadsuk, T.; Promarak, V.; Keawin, T. \& Jungsuttiwong, S. - J. Therm. Anal. Calorim., 103, p.1017 (2011). http://dx.doi.org/10.1007/ s10973-010-1022-3

16. Chen, S.; Jin, J. \& Zhang, J. - J. Therm. Anal. Calorim., 103, p.229 (2011). http://dx.doi.org/10.1007/s10973-010-0957-8

17. Jin, J., Chen, S. J. \& Zhang, J. - Express Polym Lett., 4, p.141 (2010). http://dx.doi.org/10.3144/ expresspolymlett.2010.19

18. Guimarães, L. M. \& Zanotto, E. D. - Quim. Nova, 26, p.202 (2003).

19. Zou, P.; Tang, S. W.; Fu, Z. Z. \& Xiong, H. G. - Int. J. Therm. Sci., 48, p.837 (2009). http://dx.doi.org/10.1016/j. ijthermalsci.2008.06.010

20. Mastai, Y.; Diamant, Y.; Aruna, S. T.; \& Zaban, A. - Langmuir, 17, p.7118 (2001). http://dx.doi. org/10.1021/la010370g

21. Brandt, K.; Salikov, V.; Özcoban,, H.; Staron, P.; Schreyer, A.; Prado, L. A. S. A.; Schulte, K.; Heinrich, S. \& Schneider, G. A. - Compos. Sci. Technol., 72, p.65 (2011). http://dx.doi.org/10.1016/j.compscitech.2011.10.001

22. Ferreira, E. K. - "Estudo dos pigmentos e suas aplicações em materiais termoplásticos", Monografia, Faculdade de Tecnologia de Sorocaba, São Paulo, Brasil (2012).

23. Broda, J. - Polymer, 44, p.1619 (2003). http://dx.doi. org/10.1016/S0032-3861(02)00934-5

Enviado: Nov. 11, 2013 Reenviado: Mar. 12,2014 Aceito: Abr. 15, 2014 